\title{
AVALIAÇÃO DO AMBIENTE DE APRENDIZAGEM WEBPROINTER: CONCEPÇÃO E DESENVOLVIMENTO DE UMA FERRAMENTA WEB
}

Silvio Henrique Fiscarelli, Janio Itiro Akamatsu ${ }^{1}$

\section{Resumo}

Este trabalho descreve as principais diretrizes utilizadas no desenvolvimento do Módulo de Acompanhamento e Avaliação, do ambiente colaborativo para desenvolvimento de projetos interdisciplinares.- WebProInter .Os principais objetivos do sistema de avaliação são: subsidiar os pesquisadores para o planejamento e a tomada de decisões; conhecer em profundidade os pontos fortes e fracos do ambiente; contribuir para a definição de prioridades de ação; possibilitar a redefinição de objetivos e ações, quando necessário; e produzir um sistema de informações quantitativas e qualitativas para o acompanhamento da trajetória de desenvolvimento dos projetos e aprendizagem dos alunos envolvidos. Os resultados obtidos a partir da pesquisa fundamentaram-se em três aspectos: a) Interatividade; b) Desenvolvimento das atividades e produtos c) Acompanhamento contínuo de aprendizagem, considerados fundamentais para o processo de avaliação do ambiente WebProInter. Buscando captar esses aspectos foram desenvolvidas ferramentas que possibilitassem auxiliar o trabalho de análise.

\section{1 - Introdução}

Este trabalho descreve as principais diretrizes utilizadas no Desenvolvimento do Subsistema de Avaliação, concebido para ser parte integrante do "Sistema Integrado de Projetos Interdisciplinares" - WebProInter - que consiste em um ambiente educacional, colaborativo e on-line para desenvolvimento de projetos interdisciplinares. Este Sub-Sistema de Avaliação é parte de uma pesquisa de Pós-doutoramento financiada pela FAPEP (Fundação de Amparo a Pesquisa do Estado de São Paulo) que inclui não só a concepção do sub-sistema, mas também o acompanhamento e análise dos resultados obtidos com a utilização do mesmo.

O sistema WebProinter foi desenvolvido e está sendo utilizado em uma experiência piloto, denominada "Rio Paraíba: Preservando o Futuro"2, envolvendo sete escolas de Ensino Médio da região do Vale do Paraíba. Tem como princípios metodológicos a Aprendizagem por Projetos - Project Based Learning (BIE, 2003), no qual os alunos são organizados em grupos de trabalho e orientados a pesquisarem de forma cooperativa e interdisciplinar, buscando por meio de técnicas didáticas diversificadas e diferentes meios promover a interação entre os alunos participantes para a troca de informações e construção de conhecimentos sobre questões ligadas ao meio ambiente e, mais especificamente, sobre o Rio Paraíba do Sul.

A proposta de desenvolvimento do ambiente de aprendizagem colaborativo WebProInter surgiu como ferramenta que possibilita a coordenação e interação entre as escolas envolvidas no projeto. Sua principal premissa é permitir uma aprendizagem baseada na participação ativa dos educandos, vivenciando as situações-problema, refletindo sobre elas e tomando atitudes diante dos fatos.

De um modo geral, podemos dizer que um Ambiente Colaborativo de Aprendizagem é um espaço de interação que sustenta a construção, inserção e troca de informações pelos participantes, visando a construção social do conhecimento. Os ambientes colaborativos de aprendizagem têm origem em um tipo de prática denominada Trabalho Cooperativo Apoiado por Computador (Computer Supported Cooperative Work - CSCW), criado por Geif e Cashman, em

\footnotetext{
${ }^{1}$ Faculdade de Engenharia de Guaratinguetá - UNESP

Apoio: FAPESP silviohf@yahoo.com

${ }^{2}$ O sistema foi concebido e está sendo coordenado por um grupo de pesquisadores da Faculdade de Engenharia de Guaratinguetá - UNESP, e está sendo utilizado no projeto "Projetos Interdisciplinares e as Novas Tecnologias: Experiência Piloto no Ensino Médio” (Programa de Ensino Público - Fapesp).
} 
1984. Mais recentemente, a expressão "Aprendizagem Cooperativa Auxiliada por Computador" (Computer Supported Cooperative Learning - CSCL) tem sido mais utilizada e designa uma abordagem que procura ampliar a concepção do computador como uma ferramenta que pode facilitar a aprendizagem (LUCENA, 1997).

Em sua essência, um ambiente colaborativo de aprendizagem é um sistema que permite a diferentes usuários participar, colaborar ou cooperar em torno de um objetivo comum, por meio de ações, e que culminam no desenvolvimento de um produto coletivo. Diferencia-se de outros sistemas de apoio a aprendizagem porque prioriza e destaca ferramentas e suportes que promovem a colaboração.

De acordo com a teoria sócio-interacionista de Vygotsky, é na interação que surge a aprendizagem, de modo que poderíamos dizer que os conceitos teóricos deste autor são as principais fontes de inspiração para a construção desse tipo de ambientes. Neste contexto, o "mediador", que pode ser o professor ou um tutor, deve criar um clima que propicie a interação, através de atitudes de encorajamento dos alunos para o desenvolvimento das atividades, a troca de informações e o espírito de equipe. O indivíduo sente-se assim, parte integrante e ativa e, portanto, responsável pelo processo da própria aprendizagem e a do grupo, colaborando nas ações e atividades que permitem alcançar os objetivos em comum do grupo.

A avaliação de ambientes de aprendizagem informatizados envolve diversos aspectos bastante complexos e relacionados entre si; não podendo se restringir apenas aos aspectos cognitivos, mas deve englobar também aspectos comportamentais. Qualquer tentativa de avaliar e acompanhar o aprendizado do aluno, nesses ambientes envolve considerar, além do referencial teórico e metodológico da avaliação, as questões tecnológicas. Considerando a importância da avaliação, cada vez mais os ambientes desenvolvidos têm disponibilizado ferramentas que possibilitam ao professor ou outro ator responsável pela avaliação acompanhar o aluno durante a interação com o próprio ambiente e com outros usuários. O número de acessos e o tempo de permanência no Ambiente Virtual de Aprendizagem, por exemplo, apesar de representarem, a priori, uma avaliação quantitativa, se observados sob outro ponto de vista, nos fornece informações representativas no que concerne ao interesse e à participação do aluno. A avaliação em ambientes colaborativos é um processo contínuo, onde aspectos como interesse, cooperação e participação nas atividades propostas são extremamente importantes. Neste sentido, este trabalho busca apresentar e descrever a concepção do processo de avaliação e ferramentas implementadas no ambiente WebProInter.

\section{2 - Visão geral do sistema}

Para o melhor entendimento da proposta de avaliação é importante conhecer os principais recursos do sistema. O ambiente WebProInter, para efeitos de concepção e desenvolvimento, é composto por três sub-sistemas. 


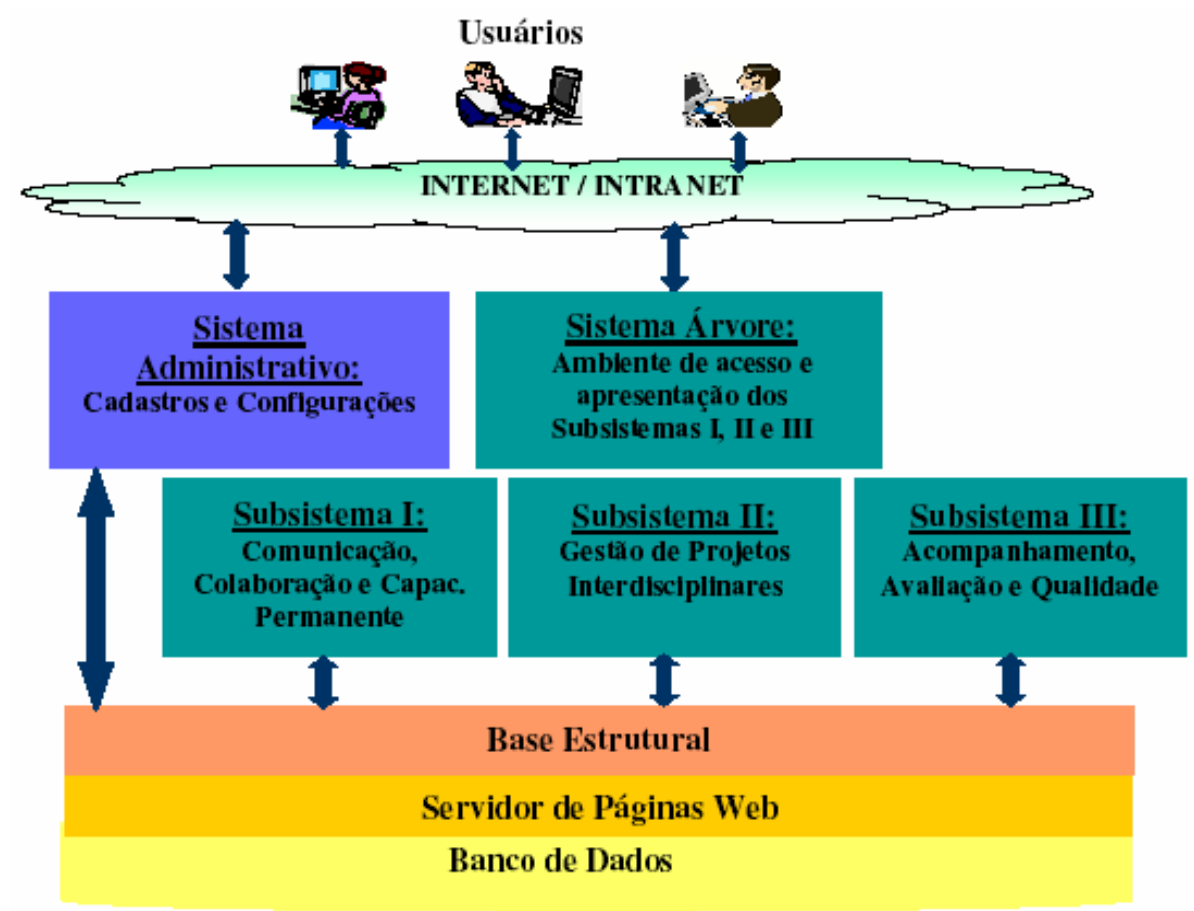

O Sub-Sistema I - Comunicação disponibiliza aos usuários ferramentas para comunicação, colaboração e integração, tais como: fórum, chats, fotologs, área de arquivos compartilhados(downloads), quadro de aviso dos grupos, permitindo a troca de diversos tipos de informações entre os usuários. As ferramentas deste sub-sistema, com pequenas variações na funcionalidade, são encontradas em todos os ambientes colaborativos.

O Sub-Sistema II - Gestão de Projetos, tratando-se de um ambiente que se fundamenta na metodologia Project Based Learning, é a principal ferramenta que possibilita o uso desta metodologia, auxilia os usuários, principalmente os coordenadores de grupos, na tarefa de elaborar, propor, acompanhar e coordenar os projetos desenvolvidos de forma mais amigável. Este sub-sistema permite, entre outras funções, coordenar equipes, atribuir tarefas, criar cronogramas de atividades, elaborar conteúdos individuais e coletivos, e relatórios sobre o desenvolvimento do projeto. A opção de criar-se uma ferramenta para o gerenciamento de projetos dos grupos surgiu justamente da necessidade de que juntamente com o produto deve existir todo um registro do processo, que será utilizado para acompanhamento pelo professor ou responsável pela avaliação.

O Sub-Sistema III: Avaliação contempla a avaliação geral do sistema, com estatísticas de acesso às ferramentas que auxiliam nas tarefas de avaliação dos resultados dos projetos desenvolvidos no ambiente, tais como: relatórios finais, resultado dos projetos, informações gerais. É a avaliação geral do sistema com estatísticas de acesso às ferramentas, aplicação de surveys, análise das atividades, avaliação do portfólio do grupo e rubricas para os produtos desenvolvidos durante a execução do projeto.

\section{3 - Avaliação}

A proposta de avaliação fundamenta-se em três aspectos que consideramos fundamentais para o sucesso do ambiente WebProInter, em desenvolvimento: a) Interatividade; b) Desenvolvimento das atividades e produtos c) Acompanhamento contínuo de aprendizagem. Buscando captar esses aspectos foram desenvolvidas, dentro do sistema, 
ferramentas que possibilitassem auxiliar o trabalho de análise. A figura 2 apresenta uma visão geral da proposta de avaliação.

Como objetivos da avaliação do ambiente Web Prointer podemos destacar os seguintes objetivos:

- Subsidiar os pesquisadores para o planejamento e a tomada de decisões;

- Conhecer em profundidade os pontos fortes e fracos do ambiente;

- Contribuir para a definição de prioridades de ação;

- Desenvolver um processo criativo de autocrítica;

- Promover a transparência das decisões e ações;

- Possibilitar a redefinição de objetivos e ações, quando necessário;

- Produzir um sistema de informações quantitativas e qualitativas para o acompanhamento da trajetória de desenvolvimento dos projetos e aprendizagem dos alunos envolvidos.

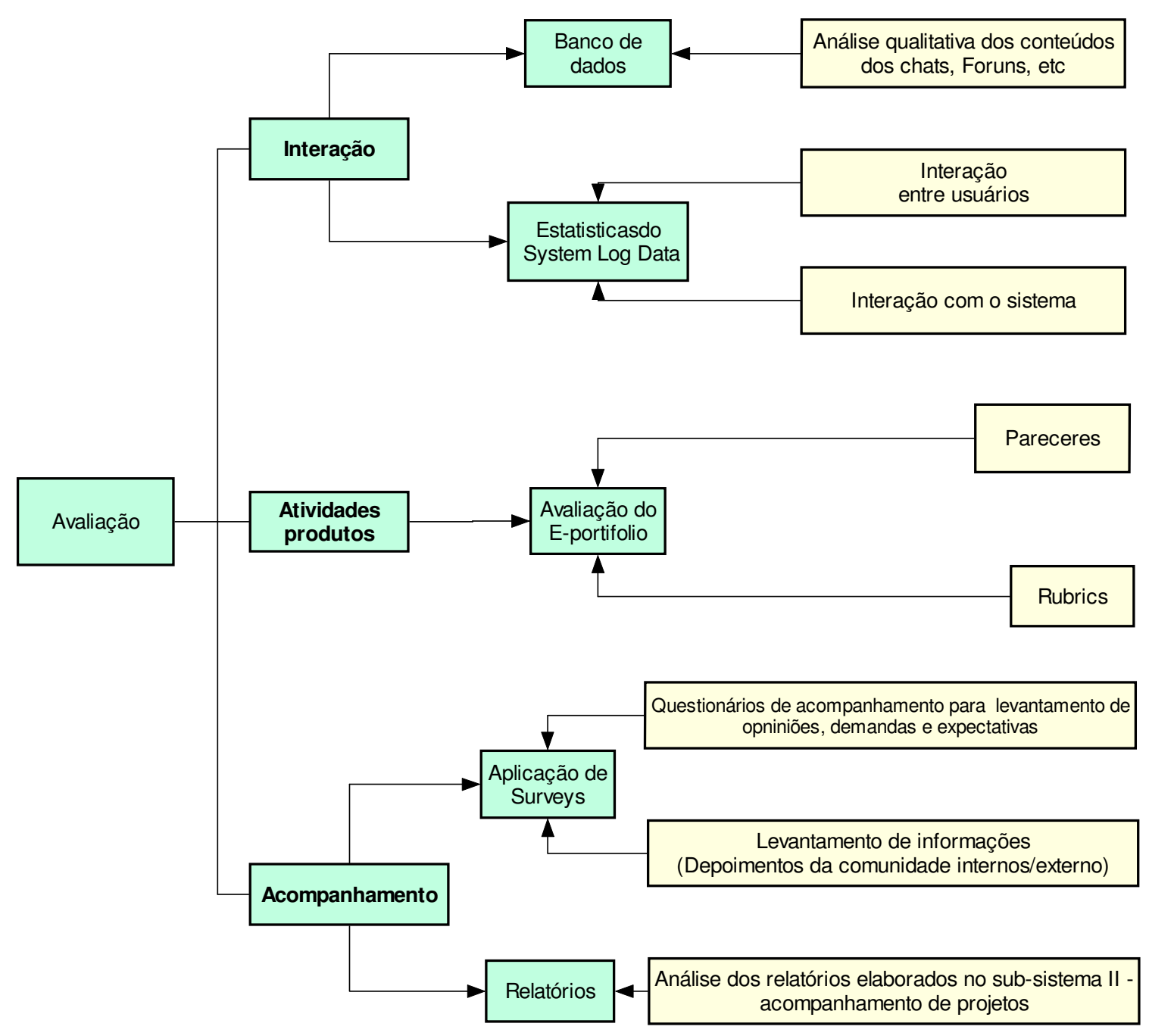

Figura 2 - Visão geral da proposta de avaliação 


\section{1- Interatividade}

A interatividade em um ambiente de aprendizagem on-line é realizada fundamentalmente pelas formas de diálogo, isto é, por meio de ferramentas de comunicação eletrônica (chats, fóruns de discussão, news, e-mail, videoconferência, etc) e outros instrumentos de interação, que em sua grande maioria são baseados na linguagem escrita. Neste contexto essas ferramentas possibilitam aos alunos trocarem informações com outros participantes, participarem de discussões, organizarem suas ações a fim de construir produtos e conhecimento de forma interativa (CAMPOS, 2003)

A avaliação da interatividade apóia-se em um referencial teórico-metodológico proveniente dos estudos sobre o uso das "Novas Tecnologias de Comunicação e Informação" na educação, conforme apontadas por Valente (1993, 1996), Feijó (1997), Fagundes (1999), Santarosa et alii (1995), Chaves (1999), Primo (1998), entre outros. Busca investigar, empiricamente, os resultados da interatividade proporcionada pelo uso de recursos informáticos de comunicação em ambientes de aprendizagem.

A tecnologia disponível hoje permite a implementação de ambientes (sistemas computacionais) com grande qualidade de interação do usuário com o software. Opções minuciosamente programadas podem levar os usuários a agir de forma criativa e fornecerem um alto grau de liberdade de ações nestes ambientes virtuais, mas esse tipo de interação talvez não seja o objetivo primordial dos ambientes cooperativos educacionais. Quando buscamos um ambiente cooperativo educacional, o principal foco está na interação entre os usuários e não somente na interação com a máquina. O termo interativo muitas vezes é utilizado apenas no sentido de permitir ao usuário interagir com os diversos conteúdos dos ambientes, mas para um sistema ser verdadeiramente interativo deve facilitar a comunicação entre os usuários (PRIMO,1998).

Para levantar indícios que permitam inferir sobre a interatividade proporcionada pelo sistema, são utilizados dois procedimentos básicos:

Análise qualitativa: todo conteúdo dos fóruns e chats produzidos pelos usuários é arquivado no banco de dados para posterior análise qualitativa de seu conteúdo. As análises dispõem de suporte para buscas textuais, a partir de palavras-chaves e incorporação de comentários atrelados a determinados conteúdos e contextos.

Análise quantitativa: refere-se ao uso de análises estatísticas de dados provenientes do que chamamos de "System Log Data" (LTDI - Evaluation Cookbook,1998). Muitos softwares, principalmente os que são executados em ambiente web, realizam, durante as operações efetuadas pelos usuários, o registro de informações sobre as operações em arquivos ou bancos de dados para fins de segurança, depuração ou mesmo posterior acompanhamento das operações realizadas. Em ciência da computação, o "System Log Data" é muito utilizado, principalmente em sistemas cliente-servidor. Por meio dele os administradores verificam a usabilidade do software e a ocorrência de erros (KRISTOL e MONTULLI, 1997). Esses dados técnicos, que inicialmente eram utilizados somente por administradores dos sistemas, começaram a ser utilizados com fins de avaliação (WHITEFIELD, A., WILSON, F. e DOWELL, J.,1991), pois guardam informações que podem fornecer características importantes sobre o usuário, os tipos de operações que realizou e mesmo sobre a interatividade entre usuários e sistema.

O "System Log Data" pode ser criado e configurado para que arquive os mais diversos tipos de dados, como o nome do usuário que está acessando o sistema; horário que realizou login; quanto tempo permaneceu utilizando o sistema; quais áreas, páginas, funções e ferramentas utilizou; bem como outras possibilidades e necessidades que forem identificadas.

Neste sentido, foi construído para o ambiente WebProInter uma ferramenta que realiza um tratamento estatístico desses dados gerados pelo "System Log Data", possibilitando ao 
avaliador acesso a um conjunto de informações previamente agregadas que facilitam a compreensão das ações e interações dos usuários no ambiente.

Para tanto, durante o desenvolvimento do sistema, medidas foram tomadas para que o mesmo disponha de um "System Log Data" com características amplas, pois, tratando-se de um sistema para fins educacionais, consideramos importante armazenar dados e informações que permitam aos pesquisadores do projeto realizarem análises mais profundas. Com o estudo quantitativo e qualitativo da interatividade do sistema WebProInter espera-se que sejam obtidos avanços para a avaliação do mesmo, pois informações preciosas como o tipo de utilização e quais formas de interações realizadas no ambiente serão de grande importância para a avaliação de resultados e impactos do sistema.

Para cada usuário do ambiente WebProInter, o System Log Data registrará:

- Data e hora de entrada no ambiente;

- Data, hora e tipo de ferramentas ou recursos que utilizou no ambiente;

- Tempo que permaneceu utilizando cada ferramenta ou recurso;

- Data e hora de saída do ambiente.

Mediante análises estatísticas e cruzamento de dados será possível obter-se as seguintes informações:

- Caracterização dos usuários (nome, grupo e escola que pertence, etc.).

- Ferramentas e recursos do ambiente mais utilizados.

- Interação síncrona (número de usuários utilizando a mesma ferramenta ou recurso simultaneamente, bem como o tempo que permaneceram interagindo na ferramenta).

- Interação assíncrona (histórico de uso das ferramentas ou recursos do ambiente, por usuário, grupo ou escola.).

- Acompanhamento de uma atividade mediante a análise do histórico de uso das ferramentas.

\subsection{Desenvolvimento de atividades e produtos}

São fornecidas no ambiente WebProInter ferramentas nas quais os alunos trabalham em projetos, problemas e temas para pesquisa, compartilhando soluções e dividindo tarefas. $\mathrm{O}$ ambiente de aprendizagem busca, neste contexto, facilitar o trabalho em grupo por meio de recursos que permitam a realização de atividades que melhorem os conhecimentos e habilidades específicas.

A partir das atividades e interações proporcionadas pelo ambiente, espera-se como resultado do processo, o desenvolvimento de "produtos". Os produtos são entendidos, neste contexto, como evidências do processo de ensino- aprendizagem; ou seja, é a materialização do trabalho dos alunos que pode se concretizar em diferentes formas como a escrita, a leitura, a discussão, apresentações orais, teatro, etc. O produto é a maneira pelo qual os alunos demonstram seu trabalho para os outros participantes do ambiente, para o professor, e são passíveis de avaliação.

Considerando-se que a maioria dos produtos desenvolvidos é arquivos de textos, apresentações eletrônicas, planilhas de cálculo e outros tipos de materiais cujo suporte é eletrônico, acreditamos que grande parte da avaliação pode ser realizada por meio dos portfólios eletrônicos dos grupos. Os portfólios dos grupos foram concebidos como um espaço para conteúdo, repositório das informações e produtos gerados pelos participantes, tais como: textos, imagens, vídeos, sons, animações; ou seja, os mais diversos tipos de dados. Tais informações estarão organizadas em níveis de acesso intercambiáveis, permitindo que os grupos tomem 
decisão sobre as condições de disponibilização. Assim, um conteúdo pode ser considerado "rascunho" (disponível somente ao grupo de trabalho) ou "compartilhado" (acessível a todos os grupos) e "publicado" (disponível em uma página do grupo na Internet).

$\mathrm{O}$ conceito e desenvolvimento dos portfólios surgiram na área das artes, que os denomina como um conjunto de trabalhos de um artista (desenhista, pintor, fotógrafo, etc.) usado para divulgação de suas produções aos clientes. Nesse caso, é um instrumento que possibilita ao artista demonstrar e comprovar os trabalhos realizados, as suas capacidades criadoras e artísticas. A idéia básica de um portfólio é usar um conjunto de documentos arquivados e organizados para evidenciar produtos, sejam eles objetos ou processos. Dependendo da área e da situação em que é usado sua elaboração é muito semelhante a elaboração de um dossiê (SELDIN, 1997).

De acordo com o American Heritage Dictionary, portfólio é um contêiner ou receptáculo portátil para guarda de materiais, tais como: papéis avulsos, fotografias ou desenhos (esboços); ou ainda, os materiais arranjados em contêineres, especialmente quando representativos de um trabalho pessoal (ou profissional).

A principal contribuição que a construção e uso dos portfólios oferece é a possibilidade de se refletir sobre um determinado processo, a partir da documentação que foi agregada nele. Não há uma metodologia correta para a construção de um portfólio, mesmo porque não há um só meio de fazê-lo ou de usar suas informações. Portanto, suas características são determinadas segundo os propósitos específicos para os quais é desenvolvido.

Centra (1994) afirma que o portfólio vem sendo apontado como uma das mais recentes contribuições para uma avaliação do ensino. Dada suas características, um portfólio pode ser um instrumento adequado para comportar informações qualitativas e quantitativas, pois um de seus principais requisitos deve ser a possibilidade de incorporar dados de diferentes fontes e naturezas, oferecendo um conhecimento mais completo.

Considerando-se que estamos tratando de um sistema on-line, o desenvolvimento de um portfólio eletrônico oferece mais perspectivas e possibilidades que a forma tradicional, com maior amplitude de alcance e facilidade de gerenciamento de informações, favorecida ainda pela interconectividade proporcionada pela informática. $\mathrm{O}$ uso de portfólios em ambiente de rede pode facilitar a produção (conjunta ou isoladamente), propiciando o intercâmbio de experiências entre os diversos usuários do sistema.

Dentre as vantagens do portfólio eletrônico destaca-se a sua maleabilidade; isto é, quem utiliza o sistema pode dispor de formas múltiplas de apresentação, consulta e visualização de informações; as quais podem ser ordenadas cronologicamente, arranjadas por tipo, por assunto ou por área de interesse. Outra característica interessante do portfólio eletrônico é a seletividade de conteúdo, que possibilita a segregação de informações mais importantes, de acordo com o uso que se pretende fazer delas, com as áreas de interesse, com os possíveis usuários dos dados, etc.

Com base nos usos, conceitos e definições explicitadas, consideramos a sistemática adotada na construção de portfólios eletrônicos ideal para a avaliação da aprendizagem dos grupos de trabalho gerenciados pelo ambiente WebProInter. Para a avaliação do portfólio os professores devem realizar pareceres e rubricas sobre as atividades e conteúdos desenvolvidos pelos grupos. O parecer consiste em uma opinião fundamentada de caráter qualitativo sobre cada item do portfólio, salientando as evidências de aprendizagem; enquanto as rubricas possuem um caráter mais objetivo.

O professor, ao avaliar o portfólio por meio da análise e elaboração de um parecer, deve ficar atento no sentido de evidenciar, no material colecionado, subsídios para a avaliação do aluno. Esses subsídios podem ser reflexões e impressões sobre a disciplina ou curso, opiniões, dúvidas, dificuldades, reações aos conteúdos e aos textos indicados, às técnicas de ensino, sentimentos, situações vividas nas relações inter pessoais e outros aspectos. As anotações, rascunhos e materiais utilizados para realizar as diversas atividades propostas durante o ano letivo fornecem uma imagem em movimento contínuo, identificando o percurso realizado pelos alunos. 
Segundo Andrade (2000), rubricas são instrumentos particularmente úteis para avaliação de objetos complexos e subjetivos. As rubricas são criadas a partir de uma lista de critérios desejáveis para uma determinada atividade ou produto que são julgados a partir de uma escala de avaliação. Para efeitos de uma análise mais objetiva as rubricas podem conter uma coluna chamada scoring (pontuação), na qual são colocados os valores correspondentes às variáveis da escala de avaliação. Ao final do preenchimento, são somados os pontos de cada critério e obtido um resultado quantitativo (ANDRADE,2000). Esse tipo de procedimento é útil para facilitar a comparação temporal de um aluno ou grupo.

Considerada como uma forma de estabelecer pré-requisitos para a "análise" das atividades ou produtos, as rubricas podem ser utilizadas como um documento de registro, que o professor preenche e arquiva, inicialmente para efeitos de avaliação imediata, mas que, posteriormente, possibilitará acompanhar temporalmente a performance do grupo ou aluno. Algumas vantagens mais comuns atribuídas ao uso de rubricas são:

- Permitir que a análise seja mais objetiva e consistente;

- Ajudar o professor a estabelecer critérios de avaliação;

- Mostrar ao aluno como o seu trabalho será avaliado e o que se espera dele;

- Oferecer um feedback útil a respeito da eficácia do ensino;

- Oferecer uma ferramenta para medir e documentar os progressos.

\section{3 - Acompanhamento contínuo}

Buscando viabilizar o acompanhamento contínuo do projeto, foi concebida uma ferramenta on-line que facilitasse a aplicação de surveys nos usuários nele envolvidos. As pesquisas de surveys são normalmente descritas como a obtenção de dados ou informações sobre características, atitudes, opiniões, comportamento de determinado grupo de pessoas por meio de um instrumento de pesquisa, normalmente um questionário. (TANUR apud PINSONNEAULT \& KRAEMER, 1993).

Fink (1995a; 1995b) aponta que o método de pesquisa utilizando-se surveys pode ser de grande utilidade quando o interesse é produzir descrições quantitativas de uma população, e se aplicado criteriosamente apresenta elevada confiabilidade.Os surveys são adequados quando:

- se deseja responder questões do tipo "o quê?", "por quê?", “como?” e "quanto?"; ou seja, quando o foco de interesse é sobre "o que está acontecendo" ou "como e por que isso está acontecendo";

- não se tem interesse ou não é possível controlar as variáveis dependentes e independentes;

- o ambiente natural é a melhor situação para estudar o fenômeno de interesse.

A utilização de surveys junto aos usuários do ambiente Web Prointer pode, e deve, fazer parte do processo de avaliação, pois em algumas situações é de grande importância saber as atitudes e opiniões dos sujeitos envolvidos em uma situação ou objeto de avaliação. Através da aplicação de questionário, temos a oportunidade de colher informações sobre o ambiente, sobre os projetos desenvolvidos nele e demais atividades envolvidas no processo de ensinoaprendizagem. Podemos, por exemplo, usar os surveys para levantar aspectos deficitários, problemas e mesmo questões relativas à percepção do ambiente pelos usuários envolvidos.

Neste sentido, o ambiente é dotado de um uma ferramenta que possibilita a elaboração e aplicação de questionários em todos os segmentos usuários do ambiente. Esta ferramenta pode ser utilizada não só pelos coordenadores do projeto, mas também pelos professores, com o intuito de colher informações sobre os alunos.

Para o acompanhamento do projeto "Rio Paraíba: Preservando o Futuro" foram selecionados os fatores considerados essenciais para avaliação e a partir deles, elaborados indicadores e itens de avaliação. 


\section{4 - Considerações finais}

Para Woodbine (1997), a avaliação em ambientes colaborativos tem acompanhado a tendência de avaliar não somente o produto, mas todo o conjunto. Neste contexto, é preciso definir o método de avaliação (o que será avaliado), os objetivos instrucionais (o que o aluno precisa apreender) e a melhor forma de acompanhamento desse aluno (processo).

Durante as discussões entre os pesquisadores envolvidos no projeto, apareceram como principais elementos do sistema de avaliação, a necessidade de se verificar a interação proporcionada e realizada no ambiente; o processo de gerenciamento dos projetos e, conseqüentemente, os produtos gerados nesse processo, os quais são armazenados e sistematizados no sub-sistema de gestão de projetos; e finalmente a importância de se contar com um mecanismo de feedback, implementado por meio da ferramenta de survey. Durante o desenvolvimento do sub-sistema de avaliação, a preocupação dos pesquisadores voltou-se para conceber e desenvolver mecanismos que preservassem evidências das diversas formas de utilização do sistema pelos usuários.

O princípio de utilização de estatísticas sobre os dados do System Log Data, para a averiguação da interatividade entre os alunos do ambiente colaborativo, está ligado ao fato de ser possível otimizar o trabalho do professor ou responsável pela avaliação, pois receberá do sistema um conjunto de informações já agregadas e sistematizadas o que facilitará muito o processo de interpretação dos resultados. Uma análise mais profunda da interatividade pode ser realizada fazendo se busca e seleção dos dados dos chats, foruns e arquivos que ficam registrados com total integridade no sistema.

$\mathrm{Na}$ ferramenta de gestão de projeto, as atividades dos grupos são estabelecidas e definidas conforme suas necessidades. O estabelecimento de calendário de atividades e cronogramas para execução das atividades organiza o trabalho em grupo. A ferramenta para construção de textos permite a construção coletiva e colaborativa de produtos por meio de contribuições individuais de cada membro do grupo, bem como o compartilhamento de informações, uma vez que todo o material produzido é visível para todos os membros do grupo ou mesmo para membros de outros grupos, desde que configurada esta opção. A possibilidade de consulta às versões anteriores, permite que sejam feitas comparações, estimulando a reflexão sobre as contribuições. Do ponto de vista da avaliação, esta poderá ser feita não somente dentro do texto, mas também a participação individual de cada um poderá ser avaliada quantitativamente e qualitativamente, através da visualização de suas contribuições.

Tais considerações, realizadas até o presente momento, nos levaram a acreditar que a proposta de avaliação descrita e detalhada no corpo deste trabalho caracteriza-se como um sistema de informação de apoio à avaliação, não somente do ambiente Web ProInter mas que pode ser útil para outros ambientes educacionais on-line. A relação estabelecida entre o referencial teórico adotado e o acompanhamento do projeto em seu lócus de execução, permitiram-nos um olhar um pouco mais sensível para as particularidades e necessidades específicas desses tipos de ambientes educacionais.

É importante enfatizarmos que a eventual utilização das ferramentas de avaliação propostas para projetos exigirá de seus gestores posturas e concepções democráticas e participativas, pois o software é apenas um instrumento de apoio que por si só não é capaz de garantir que os processos avaliativos sejam conduzidos com ética e responsabilidade. Portanto, mais importante do que a utilização do software é a postura da comunidade envolvida no projeto frente às questões filosóficas, culturais e sociais que envolvem todo o processo de avaliação.

\section{5- Referências}

Andrade, H. G.(2000).Using rubrics to promote thinking and learning. Educational Leadership (57). 
BIE Handbook: Buck Institute of Education ( 2003). Project based learning: A Guide to standards-focused project based learning for middle and high school teachers. Oakland, CA,

Campos, Gilda (2001, Abril). "Como avaliar um Software Educacional?" Disponível em: http://www.timaster.com.br. [consulta 2005, 28 de maio].

Centra, J.(1994).The use of the teaching portfolio and student evaluation for summative evaluation. Journal of Higher Education (11-12).

Chaves E. (1999).Tecnologia na Educação, Ensino a Distância, e Aprendizagem Mediada pela Tecnologia: Conceituação Básica. Revista Educação da Faculdade de Educação da Pontifícia Universidade Católica de Campinas. Ano III, ( 7) 22-28.

Fagundes, L. C.; Sato, L. S.; Maçada, D. L (1999). Aprendizes do Futuro: as inovações começaram! Coleção Informática para a mudança na Educação. MEC/Brasil.

Feijó, L.M. (1997). Ambientes distribuídos de aprendizagem: teoria e prática da avaliação de experiência desenvolvida em sala de aula. Anais do VIII Simpósio Brasileiro de Informática na Educação - SBIE. São Paulo.

Ferreira, T. B. (2001). Desenvolvimento de uma Ferramenta de Suporte à Avaliação em Ambientes de Ensino a Distância. Dissertação de Mestrado, IC/Unicamp, 2001.

Fink, Arlene (1995a). The survey handbook . Thousand Oaks, Sage, [The Survey Kit, v.1]

. (1995b). How to design surveys. Thousand Oaks, Sage, [The Survey Kit, v.5]

Hernández, Fernando \& Ventura, Montserrat.(1998). A organização do currículo por projetos de trabalho. Porto Alegre: Artmed.

Kraemer, K.L.; Cash Jr., J.I.; Nunamaker, J.F.(1991). The information systems research challenge:survey research methods. Boston: Harvard Business School, v.3.

KRISTOL, D. \& MONTULLI, L.(1997). “HTTP State Management Mechanism”. Disponível em: http://www.ietf.org/rfc/rfc2109.txt. [consulta 2005, 11 de maio].

Lucena, Marisa. Um modelo de escola aberta na internet: KIDLINK no Brasil. Braspot: Rio de Janeiro, 1997.

Pinsonneault, A. \& Kraemer, K. L (1993). Survey research in management information systems: an assessement.Journal of Management Information System. 5-8.

Primo, A. (1998). Interação mútua e reativa: uma proposta de estudo. Anais XXI CONGRESSO DA INTERCOM, Recife, PE,

Santarosa, L.M.C; Ortolan, Adriana; Barrionuevo, Luis Otávio; Paul, Klaus.(1995) Ambiente multimídia/hipermídia no desenvolvimento cognitivo e construção da leitura e escrita. VI Simpósio Brasileiro de Informática na Educação. Florianópolis/SC, pp. 363-374, nov./95

Seldin, P. (1997).The teaching portfólio: a practical guide to improved performance and promotion / tenure decisions. Boston, MA: Anker Publishing Company.

Valente, José Armando. (1993). Diferentes Usos do Computador na Escola. Em Aberto. Brasília. (12) 3-16.

Valente, José Armando. (1997). O uso inteligente do computador na educação. Pátio. Porto Alegre.(1)10-21.

Vygotsky, L. S. (1998). A formação social da mente: o desenvolvimento dos processos psicológicos superiores. São Paulo, Martins Fontes.

Whitefield, A., Wilson, F. and Dowell, J. (1991). “A framework for human factors Mechanism”, RFC 2109 Disponível em: ftp://ftp.isi.edu/in-notes/rfc2109.txt. [consulta 2005, 12 de setembro].

Woodbine, G.(1997) Can the various forms of cooperative learning techniques be applied affectively in the classroom in content driven accounting courses. Anais of the $\boldsymbol{6}^{\text {th }}$ anual teaching learnin forum. 